Pacific

Journal of

Mathematics

SOME REMARKS ON THE PRESCRIBED MEAN CURVATURE EQUATION ON COMPLETE MANIFOLDS

Stefano Pigola, Marco Rigoli, and Alberto G. Setti 


\title{
SOME REMARKS ON THE PRESCRIBED MEAN CURVATURE EQUATION ON COMPLETE MANIFOLDS
}

\author{
Stefano Pigola, Marco Rigoli, and Alberto G. Setti \\ Dedicated to the memory of Franca Burrone Rigoli
}

\begin{abstract}
We obtain conditions on the behavior at infinity of the mean curvature of a graph under volume growth assumptions. An $L^{q}$ comparison result is also given.
\end{abstract}

\section{Introduction.}

Let $(M,\langle\rangle$,$) be a complete (noncompact), m$-dimensional, $m \geq 2$, Riemannian manifold and, for a fixed reference point $o \in M$, set $r(x)=$ $\operatorname{dist}_{(M,\langle,\rangle)}(o, x)$. Thus $B_{R}$ and $\partial B_{R}$ denote, respectively, the geodesic ball and sphere of radius $R$ centered at $o$. In what follows we shall always assume $M$ connected.

We associate to a smooth function $u: M \rightarrow \mathbb{R}$ its graph $\Gamma_{u}: M \rightarrow M \times \mathbb{R}$, defined by

$$
\Gamma_{u}: x \rightarrow(x, u(x)) .
$$

Indicating with $($, ) the product metric on $M \times \mathbb{R}$,

$$
\Gamma_{u}:\left(M, \Gamma_{u}^{*}(,)\right) \rightarrow(M \times \mathbb{R},(,))
$$

becomes an isometric embedding. Let $\nabla$, div, || denote the gradient, the divergence operator and the norm with respect to $\langle$,$\rangle . Then, \Gamma_{u}$ has mean curvature $\frac{1}{m} a(x)$, for some function $a \in \mathcal{C}^{\infty}(M)$ (and appropriately chosen normal to $\Gamma_{u}$ ) if and only if

$$
\operatorname{div}\left(\frac{\nabla u}{\sqrt{1+|\nabla u|^{2}}}\right)=a(x), \quad \text { on } M .
$$

Thus, if $a(x)$ is constant, $\Gamma_{u}$ has constant mean curvature and if $a(x) \equiv 0$, $\Gamma_{u}$ is a minimal graph (and $u$ is a minimal map).

When $M=\mathbb{R}^{m}$ is the Euclidean space, a result of S. Bernstein [B1], [B2] for surfaces (later improved in varying ways by E. Heinz $[\mathbf{H}]$, by S.S. Chern $[\mathbf{S}]$, and by R. Finn $[\mathbf{F}]$ ) implies that a graph with constant mean curvature defined on all of $M=\mathbb{R}^{m}$ is necessarily minimal. We point out that at the heart of these arguments there are estimates for the mean curvature in terms of isoperimetric quantities. 
This was later generalized to the case of graphs over Riemannian manifolds by I. Salavessa $[\mathbf{S}]$, who showed that if $\Gamma$ is a parallel mean curvature graph over $(M,\langle\rangle$,$) , then$

$$
\left\|H_{\Gamma}\right\| \leq \frac{h(M)}{m},
$$

where $h(M)$ is the Cheeger constant of $M$, and $\left\|H_{\Gamma}\right\|$ the (constant) length of the mean curvature vector of $\Gamma$. Now, if $M$ has subexponential volume growth, then $h(M)=0$, and $\Gamma$ is a minimal graph.

When $M$ has a faster volume growth there might exist non-minimal constant mean curvature graphs, as the following example shows. Let $\mathbb{H}^{m}$ be the hyperbolic space with canonical metric $\langle$,$\rangle of constant negative curva-$ ture -1 , which we realize, in polar coordinates $(r, \theta) \in(0,+\infty) \times S^{m-1}$, as

$$
\langle,\rangle=d r^{2}+(\sinh r)^{2} d \theta^{2},
$$

$d \theta^{2}$ being the standard metric on $S^{m-1}$. Then, for any $a \in(0, m-1]$ the smooth function

$$
u(x)=\int_{0}^{r(x)} \frac{(\sinh t)^{1-m} \int_{0}^{t} a(\sinh s)^{m-1} d s}{\left\{1-(\sinh t)^{2(1-m)}\left[\int_{0}^{t} a(\sinh s)^{m-1} d s\right]^{2}\right\}^{\frac{1}{2}}} d t
$$

realizes a graph $\Gamma_{u}$ with constant mean curvature $\frac{a}{m}$. In this example, note that

$$
h\left(\mathbb{H}^{m}\right)=m-1 \quad \text { and } \quad u(x) \sim r(x), \text { as } r(x) \rightarrow+\infty .
$$

Thus, in order to obtain a result similar to that of Bernstein mentioned above when $(M,\langle\rangle$,$) grows at most exponentially, it seems natural to require$ some growth conditions on $u$. Indeed, if we assume that $u(x)=o(r(x))$ as $r(x) \rightarrow+\infty$, then the constant mean curvature graph $\Gamma_{u}$ is minimal. We refer to $[\mathbf{R S V}]$ for a precise statement and details.

In this note we prove:

Theorem A. Let $\Gamma_{u}: M \rightarrow M \times \mathbb{R}$ be a graph such that

$$
\sup _{M}|u|<+\infty
$$

and with mean curvature $a(x) \in C^{\infty}(M)$ of constant sign. Assume that $(M,\langle\rangle$,$) satisfies one of the following growth assumptions:$

$$
\begin{aligned}
& \operatorname{vol}\left(\partial B_{r}\right) \leq C r^{\alpha}, \quad \text { for some } \alpha \geq 0, \\
& \operatorname{vol}\left(\partial B_{r}\right) \leq C e^{\alpha r}, \quad \text { for some } \alpha>0, \\
& \operatorname{vol}\left(\partial B_{r}\right) \leq C e^{\alpha r^{2}}, \quad \text { for some } \alpha>0,
\end{aligned}
$$


for some constant $C>0$. Then, corresponding to cases (i), (ii), (iii) we respectively have:

$$
\begin{aligned}
& \liminf _{r(x) \rightarrow+\infty} \frac{|a(x)|}{r(x)^{-2}[\log r(x)]^{-1}}=0, \\
& \liminf _{r(x) \rightarrow+\infty} \frac{|a(x)|}{r(x)^{-1}[\log r(x)]^{-1}}=0, \\
& \liminf _{r(x) \rightarrow+\infty} \frac{|a(x)|}{[\log r(x)]^{-1}}=0 .
\end{aligned}
$$

Note that if (iii) holds then (jjj) implies that a constant mean curvature graph satisfying (0.1) is minimal.

In Section 2 we describe examples which show that Theorem A is fairly sharp.

Many results in the study of assigned mean curvature graphs, rely on comparison-type properties and Theorem A is no exception (see Corollary 1.2). Loosely speaking, given a domain $\Omega$ suppose that $u$ and $v$ satisfy

$$
\begin{cases}\text { i) } \quad \operatorname{div}\left(\frac{\nabla u}{\sqrt{1+|\nabla u|^{2}}}\right) \geq \operatorname{div}\left(\frac{\nabla v}{\sqrt{1+|\nabla v|^{2}}}\right), & \text { on } \Omega \\ \text { ii) } \quad u \leq v, & \text { on } \partial \Omega .\end{cases}
$$

We look for conditions ensuring the possibility of extending inequality (0.2) ii) to all of $\Omega$.

There is a vast literature on the subject ranging from the geometrical to the analytical approach. We limit ourselves to quote the papers $[\mathbf{C K}]$, $[\mathbf{H w}],[\mathbf{H w} \mathbf{1}],[\mathbf{M}]$ which are closely related with the results below. Our contribution is twofold. In Theorem 1.1 we analyze the case where the strict inequality holds in (0.2) i), while in Theorem 1.3 we prove an $L^{q}$-comparison result from which the next uniqueness statement follows at once.

Theorem B. Let $\Omega \subseteq(M,\langle\rangle$,$) be a domain with (possibly empty) boundary$ $\partial \Omega$. Let $u, v \in \mathcal{C}^{2}(\Omega) \cap \mathcal{C}^{0}(\bar{\Omega})$, be such that

$$
\begin{cases}\operatorname{div}\left(\frac{\nabla u}{\sqrt{1+|\nabla u|^{2}}}\right)=\operatorname{div}\left(\frac{\nabla v}{\sqrt{1+|\nabla v|^{2}}}\right), & \text { on } \Omega \\ u=v, & \text { on } \partial \Omega .\end{cases}
$$

Assume that, for some $q>1$,

$$
\int_{B_{r} \cap \Omega}|u-v|^{q}=O\left(r^{2} \log r\right), \quad \text { as } r \rightarrow+\infty .
$$

Then, if $\partial \Omega \neq \varnothing, u=v$ on $\Omega$, otherwise $u=v+A$ on $M$ for some $A \in \mathbb{R}$.

The sharpness of Theorem 1.3 (and thus of Theorem B) is discussed in Section 2. 
For further details and related properties, for instance the case $q=1$, we refer the reader to Section 1.

We would like to stress that in all the results presented in the sequel, we do not impose curvature restrictions on the underlying manifold $(M,\langle\rangle$,$) .$

We use the variable constant convention and denote with $C$ a positive constant whose actual value may vary from place to place.

Acknowledgments. The authors are grateful to the anonymous referee for a very careful reading and several suggestions that led to an improvement of the paper.

\section{Proof of the results.}

The proof of Theorem 1.1 below is a variation of some ideas of Grigor'yan originally used, in the linear setting, to guarantee stochastic completeness, $[\mathbf{G}]$, and, later, $[\mathbf{G} \mathbf{1}]$, to show the non-existence of nontrivial bounded solutions of the Schrödinger equation $\Delta u-b(x) u=0, b(x) \geq 0, b(x) \not \equiv 0$, on complete manifolds. We provide details because of some differences and for the sake of completeness. First, we recall the following inequality due to Mikljukov, [M], p. 265, Hwang [Hw], p. 342, and Collin and Krust [CK], p. 452 , that we shall use below and in Lemma 1.2. For $u, v \in \mathcal{C}^{1}(\Omega)$,

$$
\begin{aligned}
\left\langle\frac{\nabla u}{\sqrt{1+|\nabla u|^{2}}}-\frac{\nabla v}{\sqrt{1+|\nabla v|^{2}}}, \nabla u-\nabla v\right\rangle & \geq \sigma(x)\left|\frac{\nabla u}{\sqrt{1+|\nabla u|^{2}}}-\frac{\nabla v}{\sqrt{1+|\nabla v|^{2}}}\right|^{2} \\
& \geq\left|\frac{\nabla u}{\sqrt{1+|\nabla u|^{2}}}-\frac{\nabla v}{\sqrt{1+|\nabla v|^{2}}}\right|^{2}
\end{aligned}
$$

where, for the ease of notation, we have set

$$
\sigma(x)=\frac{1}{2}\left[\sqrt{1+|\nabla u|^{2}}+\sqrt{1+|\nabla v|^{2}}\right](x) .
$$

Theorem 1.1. Let $\Omega \subseteq(M,\langle\rangle$,$) be an unbounded domain with (possibly$ empty) boundary $\partial \Omega$. Let $p:[0,+\infty) \rightarrow[0,+\infty)$ be a continuous function such that, for some $\bar{R}>0$ and for each $R \geq \bar{R}$, either one of the following conditions is satisfied:

$$
\frac{e^{D\left\{\int_{0}^{R} \sqrt{p(s)} d s\right\}^{2}}}{\operatorname{vol}\left(\partial B_{R} \cap \Omega\right)} \notin L^{1}(+\infty),
$$

for some constant $D>0$;

$$
\frac{\left\{\int_{R}^{\frac{3}{2} R} \sqrt{p(s)} d s\right\}^{2}}{R \log \operatorname{vol}\left(B_{2 R} \cap \Omega\right)} \geq h(R) \notin L^{1}(+\infty) .
$$


where the function $h:[\bar{R},+\infty) \rightarrow(0,+\infty)$ is continuous and monotonic non-increasing.

Let $u, v \in \mathcal{C}^{1}(\bar{\Omega}) \cap \mathcal{C}^{2}(\Omega)$ satisfy

$$
\left\{\begin{aligned}
& \text { i) } \operatorname{div}\left(\frac{\nabla u}{\sqrt{1+|\nabla u|^{2}}}\right)-\operatorname{div}\left(\frac{\nabla v}{\sqrt{1+|\nabla v|^{2}}}\right)=q(x) \geq p(r(x)), \quad \text { on } \Omega \\
& \text { ii) } \sup _{\bar{\Omega}}(u-v)<+\infty .
\end{aligned}\right.
$$

- If $\partial \Omega \neq \emptyset$ and $u \leq v$, on $\partial \Omega$, then $u \leq v$ on $\Omega$.

- If $\partial \Omega=\emptyset$, i.e., $\Omega=M$, and $q(x) \equiv 0$, then either $u \leq v$ on $M$, or $u-v$ is constant.

- If $\partial \Omega=\emptyset$ and $q(x) \not \equiv 0$, then there are no solutions to (1.4) i) satisfying (1.4) ii).

Proof. Let $u, v$ be solutions of (1.4) i) and ii). We consider first the case where $q(x) \not \equiv 0$ and assume by contradiction that there exists $x_{0}$ in the support $\operatorname{Supp}(q)$ of $q$ such that

$$
u\left(x_{0}\right)>v\left(x_{0}\right) .
$$

We set $\gamma=\sup _{\bar{\Omega}}(u-v)$ so that $0<\gamma<+\infty$. We introduce a new parameter $t \in[0,+\infty)$ and a new function $w$ on $\bar{\Omega} \times[0,+\infty)$,

$$
w(x, t)=e^{t}[u(x)-v(x)]-\gamma .
$$

We note that

$$
\text { i) } w(x, 0) \leq 0 ; \quad \text { ii) } \frac{\partial w}{\partial t}=e^{t}(u-v) ; \quad \text { iii) } \nabla w=e^{t}(\nabla u-\nabla v) .
$$

Given $\bar{T}>0$, let $g: M \times[0, \bar{T}) \rightarrow \mathbb{R}$ and $\psi: M \rightarrow \mathbb{R}$, be a Lipschitz, respectively, a compactly supported Lipschitz function, to be chosen later. Finally, for ease of notation we write $\varphi(t)=\left(1+t^{2}\right)^{-\frac{1}{2}}$ for $t \geq 0$. Denoting $w_{+}=\max \{0, w\}$, let $Z$ be the Lipschitz vector field defined by

$$
Z=\gamma \psi^{2} w_{+}^{2} e^{g+t}[\varphi(|\nabla u|) \nabla u-\varphi(|\nabla v|) \nabla v], \quad \text { on } \bar{\Omega} .
$$

We compute its divergence and we use (1.4), $u-v \leq \gamma, p \geq 0$, (1.7) iii), $\gamma>0$ to obtain

$$
\begin{aligned}
\operatorname{div} Z \geq & \psi^{2} w_{+}^{2} e^{g+t} q(x)(u-v) \\
& +2 \gamma \psi w_{+}^{2} e^{g+t}\langle\varphi(|\nabla u|) \nabla u-\varphi(|\nabla v|) \nabla v, \nabla \psi\rangle \\
& +2 \gamma \psi^{2} w_{+} e^{g+2 t}\langle\varphi(|\nabla u|) \nabla u-\varphi(|\nabla v|) \nabla v, \nabla u-\nabla v\rangle \\
& +\gamma \psi^{2} w_{+}^{2} e^{g+t}\langle\varphi(|\nabla u|) \nabla u-\varphi(|\nabla v|) \nabla v, \nabla g\rangle .
\end{aligned}
$$


Using the Schwarz and the Mikljukov-Hwang-Collin-Krust inequalities we obtain

$$
\begin{aligned}
\operatorname{div} Z \geq & \psi^{2} w_{+}^{2} e^{g+t} q(x)(u-v) \\
& -2 \gamma|\psi| w_{+}^{2} e^{g+t}|\varphi(|\nabla u|) \nabla u-\varphi(|\nabla v|) \nabla v||\nabla \psi| \\
& -\gamma \psi^{2} w_{+}^{2} e^{g+t}|\varphi(|\nabla u|) \nabla u-\varphi(|\nabla v|) \nabla v||\nabla g| \\
& +2 \gamma \psi^{2} w_{+} e^{g+2 t}|\varphi(|\nabla u|) \nabla u-\varphi(|\nabla v|) \nabla v|^{2} .
\end{aligned}
$$

We note that, by definition of $w, Z=0$ on $\partial \Omega$ for every $t$ in $[0, \bar{T})$ and that $\operatorname{supp} Z \subset \subset \Omega$ if $t>0$. Given $0 \leq T_{1}<T_{2} \leq \bar{T}$, we integrate over $\Omega \times\left[T_{1}, T_{2}\right]$ and use the divergence theorem and (1.7) ii) to deduce

$$
\begin{aligned}
& \int_{\Omega} \int_{T_{1}}^{T_{2}} \psi^{2} w_{+}^{2} e^{g} q \frac{\partial w}{\partial t} \\
& \leq-\int_{T_{1}}^{T_{2}} \int_{\Omega} 2 \gamma \psi^{2} w_{+} e^{g+2 t}|\varphi(|\nabla u|) \nabla u-\varphi(|\nabla v|) \nabla v|^{2} \\
& \quad+\int_{T_{1}}^{T_{2}} \int_{\Omega} 2 \gamma|\psi| w_{+}^{2} e^{g+t}|\varphi(|\nabla u|) \nabla u-\varphi(|\nabla v|) \nabla v||\nabla \psi| \\
& \quad+\int_{T_{1}}^{T_{2}} \int_{\Omega} \gamma \psi^{2} w_{+}^{2} e^{g+t}|\varphi(|\nabla u|) \nabla u-\varphi(|\nabla v|) \nabla v||\nabla g| .
\end{aligned}
$$

Integrating by parts with respect to $t$ on $\left[T_{1}, T_{2}\right]$ the LHS, and using the elementary inequality $2 a b \leq \varepsilon a^{2}+\varepsilon^{-1} b^{2}, a, b \geq 0, \varepsilon>0$ with appropriate choices of $\varepsilon$ on the last two terms of the RHS of the above inequality, yield

$$
\begin{aligned}
\left.\int_{\Omega} \psi^{2} w_{+}^{3} e^{g} q\right|_{T_{1}} ^{T_{2}} & \leq \int_{T_{1}}^{T_{2}} \int_{\Omega} \psi^{2} w_{+}^{3}\left\{q \frac{\partial g}{\partial t}+\frac{3}{2} \gamma|\nabla g|^{2}\right\} e^{g} \\
& +2 \int_{T_{1}}^{T_{2}} \int_{\Omega} \gamma w_{+}^{3} e^{g}|\nabla \psi|^{2}
\end{aligned}
$$

Let $R_{0}=\max \left\{\bar{R}, \operatorname{dist}_{(M,<,>)}\left(o, x_{0}\right)\right\}$ and fix $R_{2}>R_{0}$. Denoting by $C>0$ a constant to be chosen later, we define

$$
f(r)= \begin{cases}C^{-1}\left\{\int_{R_{2}}^{r} \sqrt{p(s)} d s\right\} & \text { if } r>R_{2} \\ 0 & \text { if } r \leq R_{2}\end{cases}
$$

and we let

$$
g(x, t)=\frac{f(r(x))^{2}}{t-\bar{T}}, \quad \text { for } r \geq 0, \quad 0 \leq t<\bar{T} .
$$

If $C$ in (1.9) is chosen so as to have

$$
q \frac{\partial g}{\partial t}+\frac{3}{2} \gamma|\nabla g|^{2} \leq 0
$$


then, by (1.8),

$$
\left.\int_{\Omega} \psi^{2} w_{+}^{3} e^{g} q\right|_{T_{1}} ^{T_{2}} \leq 2 \int_{T_{1}}^{T_{2}} \int_{\Omega} \gamma w_{+}^{3} e^{g}|\nabla \psi|^{2} .
$$

We fix $R_{1}>R_{2}$ and we choose $\psi$ radial satisfying

$$
|\psi| \leq 1 \text { on } M ; \quad \psi=1 \text { on } B_{R_{2}} ; \quad \psi=0 \text { off } B_{R_{1}} .
$$

Since $q(x) \geq 0, g \leq 0$ and $g(., T) \equiv 0$ on $B_{R_{2}}$,

$$
\left.\int_{\Omega} \psi^{2} w_{+}^{3} e^{g} q\right|_{T_{1}} ^{T_{2}} \geq E\left(R_{2}, T_{2}\right)-E\left(R_{1}, T_{1}\right)
$$

where we have set

$$
E(R, T)=\int_{\Omega \cap B_{R}} q(x) w_{+}(x, T)^{3} \geq 0, \quad R \geq R_{0} .
$$

With these choices of $\psi$ and $g$, inserting (1.12) into (1.10) yields

$$
E\left(R_{2}, T_{2}\right)-E\left(R_{1}, T_{1}\right) \leq 2 \gamma \int_{T_{1}}^{T_{2}} \int_{\left(B_{R_{1}} \backslash B_{R_{2}}\right) \cap \Omega}\left(\psi^{\prime}(r)\right)^{2} w_{+}^{3} e^{g} .
$$

We now distinguish two cases.

First case. Condition (1.2) is satisfied. Let $T_{2}=\bar{T}$. We use (1.13) together with (1.6) and the co-area formula to get

$$
E\left(R_{2}, T_{2}\right)-E\left(R_{1}, T_{1}\right) \leq C \gamma^{4} e^{3 T_{2}} \int_{R_{2}}^{R_{1}}\left(\psi^{\prime}(r)\right)^{2} \operatorname{vol}\left(\partial B_{r} \cap \Omega\right) e^{\frac{f(r)^{2}}{T_{1}-T_{2}}} d r
$$

valid for all radial Lipschitz functions satisfying (1.11). Letting $\psi$ be the function defined by $\psi(r)=1$ if $0 \leq r \leq R_{2}$,

$$
\psi(r)=\frac{\int_{r}^{R_{1}} \operatorname{vol}\left(\partial B_{r} \cap \Omega\right)^{-1} e^{-\frac{f(r)^{2}}{T_{1}-T_{2}}} d r}{\int_{R_{2}}^{R_{1}} \operatorname{vol}\left(\partial B_{r} \cap \Omega\right)^{-1} e^{-\frac{f(r)^{2}}{T_{1}-T_{2}}} d r}
$$

if $R_{2} \leq r \leq R_{1}$, and $\psi(r)=0$ if $r \geq R_{1}$, we obtain

$$
E\left(R_{2}, T_{2}\right)-E\left(R_{1}, T_{1}\right) \leq C \gamma^{4} e^{3 T_{2}}\left\{\int_{R_{2}}^{R_{1}} \frac{e^{-\frac{f(r)^{2}}{T_{1}-T_{2}}}}{\operatorname{vol}\left(\partial B_{r} \cap \Omega\right)} d r\right\}^{-1} .
$$

Fix $D$ such that (1.3) holds. Then there exist constants $C=C\left(R_{2}\right)>0$ and $\alpha>0$ such that

$$
\frac{f(r)^{2}}{\alpha} \geq D\left\{\int_{0}^{r} \sqrt{p(s)} d s\right\}^{2}-C .
$$


Hence, whenever we choose $0 \leq T_{1}<T_{2}$ so that $T_{2}-T_{1} \leq \alpha$, it follows from (1.14) that

$$
E\left(R_{2}, T_{2}\right) \leq E\left(R_{1}, T_{1}\right)+C \gamma^{4} e^{3 T_{2}}\left\{\int_{R_{2}}^{R_{1}} \frac{e^{D\left\{\int_{0}^{r} \sqrt{p(s)} d s\right\}^{2}}}{\operatorname{vol}\left(\partial B_{r} \cap \Omega\right)} d r\right\}^{-1} .
$$

Since $E(R, T)$ is a non-decreasing function of $R, R \geq R_{0}$, the limit

$$
\lim _{R \rightarrow+\infty} E(R, T)=E(+\infty, T)
$$

exists, and assumption (1.2), together with $T_{2}-T_{1} \leq \alpha$, (1.15) gives

$$
E\left(R_{2}, T_{2}\right) \leq E\left(+\infty, T_{1}\right) .
$$

Choosing $T_{1}=0$ and $0<T_{2} \leq \alpha,(1.7)$ i) and (1.16) yield

$$
E\left(R_{2}, T_{2}\right) \leq E(+\infty, 0)=0
$$

and, since $R_{2}>R_{0}$ is arbitrary, $E\left(R, T_{2}\right)=0$ for every $R>0$. Choosing $T_{1}=\alpha$ and $\alpha<T_{2} \leq 2 \alpha$, we obtain

$$
E\left(R_{2}, T_{2}\right) \leq E(+\infty, \alpha)=0 .
$$

Proceeding in this way we get $E(R, T)=0$ for each $T \geq 0$ and $R>0$. It follows from the definition of $E$ that $w_{+}(x, T) \equiv 0$ in a neighborhood of $x_{0}$ for each $T \geq 0$. Thus at $x_{0}$

$$
u\left(x_{0}\right)-v\left(x_{0}\right) \leq \gamma e^{-t}, \quad \text { for each } t \geq 0 .
$$

Using (1.5) we obtain a contradiction.

Second case. Suppose that Condition (1.3) holds. We choose $\tau>0, \eta \in(0, \tau]$ and we set $T_{1}=\tau-\eta, T_{2}=\tau, \bar{T}=\tau+\eta, R_{1}=2 R, R_{2}=R$, with $R>R_{0}$. Next, for every $R$, we choose a radial function $\psi=\psi_{R}$ satisfying (1.11) and such that

$$
\psi \equiv 1 \text { on } B_{3 R / 2} \quad \text { and } \quad\left|\psi^{\prime}\right| \leq \frac{C}{R},
$$

for some constant $C>0$ independent of $R$. Then, (1.13), the definitions of $w_{+}$and $\gamma$, and the fact that

$$
g(r, t) \leq-(2 C)^{-1} \frac{\left\{\int_{R}^{\frac{3}{2} R} \sqrt{p(s)} d s\right\}^{2}}{\eta}, \quad \text { on }\left[\frac{3}{2} R,+\infty\right) \times[\tau-\eta, \tau),
$$

give

(1.17) $E(R, \tau) \leq E(2 R, \tau-\eta)+\frac{c(\tau)}{R^{2}} \operatorname{vol}\left(\Omega \cap B_{2 R}\right) e^{-(2 C)^{-1} \frac{\left\{\int_{R}^{\frac{3}{2} R} \sqrt{p(s)} d s\right\}^{2}}{\eta}}$ 
where

$$
c(\tau)=c\left(e^{\tau}-1\right)^{3} \tau, \quad c>0 .
$$

We choose $\eta=\eta(R, \tau)$ so small that

i) $0<\eta \leq \tau$, ii) $\log \operatorname{vol}\left(\Omega \cap B_{2 R}\right)-(2 C)^{-1} \frac{\left\{\int_{R}^{\frac{3}{2} R} \sqrt{p(s)} d s\right\}^{2}}{\eta} \leq 0$. According to (1.2), this holds provided

$$
0<\eta \leq \min \left\{(2 C)^{-1} R h(R), \tau\right\} .
$$

Then, (1.17) gives

$$
E(R, \tau) \leq E(2 R, \tau-\eta)+\frac{c(\tau)}{R^{2}} .
$$

Let $R_{k}=2^{k} R$. Since

$$
\sum_{k=0}^{+\infty} h\left(R_{k}\right) R_{k} \geq \int_{R}^{+\infty} h(r) d r=+\infty
$$

there exists $k_{0}=k_{0}(R, \tau) \in \mathbb{N}$ such that

$$
(2 C)^{-1} \sum_{k=0}^{k_{0}} h\left(R_{k}\right) R_{k} \geq \tau
$$

We set, for $k=0, \ldots, k_{0}$,

$$
\eta_{k}=\tau \frac{h\left(R_{k}\right) R_{k}}{\sum_{j=0}^{k_{0}} h\left(R_{j}\right) R_{j}}
$$

and we define

$$
\tau_{0}=\tau ; \quad \tau_{k}=\tau-\sum_{j=0}^{k-1} \eta_{j}, k=0, \ldots, k_{0}+1 .
$$

A simple verification shows that

$$
\begin{aligned}
\text { i) } & 0<\eta_{k} \leq \min \left\{(2 C)^{-1} R_{k} h\left(R_{k}\right), \tau_{k}\right\} \\
\text { ii) } & c\left(\tau_{k}\right) \leq c(\tau) \\
\text { iii) } & \tau_{k_{0}+1}=0 .
\end{aligned}
$$

Because of (1.19) i), ii) we can use inequality (1.18) to obtain

$$
E\left(R_{k}, \tau_{k}\right) \leq E\left(R_{k+1}, \tau_{k+1}\right)+\frac{c(\tau)}{R_{k}^{2}},
$$


for each $k=0, \ldots, k_{0}+1$. Recalling (1.19) iii) and (1.7) i), we obtain

$$
0 \leq E(R, \tau) \leq E\left(R_{k_{0}+1}, 0\right)+c(\tau) \sum_{k=0}^{k_{0}} \frac{1}{R_{k}^{2}} \leq \frac{4 c(\tau)}{3} \frac{1}{R^{2}},
$$

whence, letting $R$ tend to $+\infty$,

$$
\int_{\Omega} q(x) w_{+}^{3}(x, \tau)=0 .
$$

Since $q>0$ in a neighborhood of $x_{0}$, we deduce

$$
w_{+}\left(x_{0}, \tau\right)=0
$$

that is

$$
u\left(x_{0}\right)-v\left(x_{0}\right) \leq \gamma e^{-\tau} .
$$

Since $\tau$ can be arbitrarily large, this yields the contradiction required to complete the proof that $u\left(x_{0}\right) \leq v\left(x_{0}\right)$ even in this case.

To conclude, we consider first the case where $\partial \Omega \neq \emptyset$. By what showed above, $u \leq v$ on $\operatorname{Supp}(q)$, and we need to prove that the inequality holds on the whole of $\Omega$. The authors are indebted to the referee who suggested the following argument. If (1.3) holds, then for every $R$ there exist $R<r_{1}<$ $r_{2}<3 R / 2$, such that $p(r)>0$ in $\left(r_{1}, r_{2}\right)$. By what proved above, $u \leq v$ on $\left(B_{r_{2}} \backslash B_{r_{1}}\right) \cap \Omega$. By the maximum principle, the inequality holds on $B_{r_{2}} \cap \Omega$, and, letting $R \rightarrow+\infty$, it holds on $\Omega$.

Assuming instead the validity of (1.2), we distinguish two cases. If $\sqrt{p(t)}$ is not in $L^{1}(+\infty)$, then $p\left(r_{i}\right)>0$ along a sequence $r_{i} \rightarrow+\infty$, and the conclusion follows as above. If $\sqrt{p(t)}$ is in $L^{1}(+\infty)$, then Condition (1.2) becomes

$$
\frac{1}{\operatorname{vol}\left(\partial B_{R} \cap \Omega\right)} \notin L^{1}(+\infty),
$$

and the conclusion follows from Theorem 1.6 below (whose proof is independent of Theorem 1.1).

Next, let $\Omega=M$. If $q(x) \not \equiv 0$, the argument above shows that $u \leq v$ on $M$. Since $u+A$ satisfies the same assumptions as $u$, it follows that $u+A \leq v$ for every $A$. Since this is clearly impossible, this shows that there are no solutions of (1.4) i) satisfying (1.4) ii).

Finally, if $\Omega=\emptyset$ and $q(x) \equiv 0$ on $M$, the conclusion follows from Theorem 1.6 as above.

Remark. Conditions (1.2) and (1.3) are independent of each other. To see this consider the following examples.

(1) Let $\mathbb{R}^{2} \backslash\{0\}=(0,+\infty) \times S^{1}$ be endowed with the metric

$$
\langle,\rangle_{g}=d r^{2}+g^{2}(r) d \theta^{2}
$$


where $d \theta^{2}$ is the standard metric on $S^{1}$ and $g(r)=r e^{\sqrt{1+r^{2}}-1}$. Then, $\langle,\rangle_{g}$ can be extended to a smooth, complete metric on $\mathbb{R}^{2}$. Set $(M,\langle\rangle)=,\left(\mathbb{R}^{2},\langle,\rangle_{g}\right)$. We note that, for $r>0$ sufficiently large,

$$
\operatorname{vol}\left(\partial B_{r}\right) \geq C_{1} e^{r}
$$

and

$$
\operatorname{vol}\left(B_{r}\right) \leq C_{2} e^{\alpha r}
$$

with $\alpha, C_{i}>0$ suitable constants, $i=1,2$. We choose

$$
p(s)= \begin{cases}\frac{(\log s-1)^{2}}{4 s(\log s)^{3}} & \text { for } s>e \\ 0 & \text { for } s \leq e\end{cases}
$$

and we observe that

$$
\sqrt{p(s)}=\frac{d}{d s} \sqrt{\frac{s}{\log s}}, \quad s>e .
$$

Thus, for each $D>0$ and for each $R>e$ large enough, it holds

$$
\frac{e^{D\left\{\int_{0}^{R} \sqrt{p(s)} d s\right\}^{2}}}{\operatorname{vol}\left(\partial B_{R}\right)} \leq C_{1}^{-1} e^{R\left(\frac{D}{\log R}-1\right)} \in L^{1}(+\infty),
$$

proving that Condition (1.2) is not satisfied. On the other hand,

$$
\frac{\left\{\int_{R}^{\frac{3}{2} R} \sqrt{p(s)} d s\right\}^{2}}{R \log \operatorname{vol}\left(B_{2 R}\right)} \geq C \frac{1}{R \log R}, \quad R \text { large, }
$$

for some constant $C>0$, so that, setting $h$ to denote the RHS of this latter inequality, Condition (1.3) is met.

(2) We let $(M,\langle\rangle)=,\left(\mathbb{R}^{2}\right.$, can $)$ and we define

$$
p(s)=\frac{1}{4(s+e)^{2} \log (s+e)} .
$$

Then, we have

$$
\frac{e^{\left\{\int_{0}^{R} \sqrt{p(s)} d s\right\}^{2}}}{\operatorname{vol}\left(\partial B_{R}\right)} \rightarrow \frac{e}{2 \pi} \quad \text { as } R \rightarrow+\infty
$$

proving that Condition (1.2) is met. On the other hand,

$$
\frac{\left\{\int_{R}^{\frac{3}{2} R} \sqrt{p(s)} d s\right\}^{2}}{R \log \operatorname{vol}\left(B_{2 R}\right)} \sim C \frac{1}{R(\log R)^{2}}, \quad \text { as } R \rightarrow+\infty
$$

for some constant $C>0$. 
Proof of Theorem A. Possibly defining $\widetilde{u}=-u$ we may suppose that $a(x) \geq$ 0 and, by (0.1), that $\sup _{M} \widetilde{u}<+\infty$. We first consider Case (i). Assume by contradiction that there exist $C, R>0$ such that, for $r(x) \geq R$,

$$
a(x) \geq \frac{C}{r(x)^{2} \log r(x)} .
$$

We choose $p:[0,+\infty) \rightarrow[0,+\infty)$ continuous satisfying $p(r)=C r^{-2}(\log r)^{-1}$, for $r \geq R$, and $a(x) \geq p(r(x))$, on $M$. According to (i), (1.2) holds for $D>0$ sufficiently large. A contradiction is achieved by applying Theorem 1.1.

In the remaining cases, one proceeds in a similar way, using (1.3) instead of (1.2).

Lemma 1.2. Let $\Omega \subseteq(M,\langle\rangle$,$) be an unbounded domain and let u, v \in$ $\mathcal{C}^{2}(\Omega) \cap \mathcal{C}^{0}(\bar{\Omega})$ be such that, for some constant $B$,

$$
\Omega_{B}=\{x \in \Omega: u(x)>v(x)+B\}
$$

is nonempty, with boundary $\partial \Omega_{B}$ contained in $\Omega$, and $u-v$ is nonconstant on $\Omega_{B}$. Suppose that

$$
\operatorname{div}\left(\frac{\nabla u}{\sqrt{1+|\nabla u|^{2}}}\right) \geq \operatorname{div}\left(\frac{\nabla v}{\sqrt{1+|\nabla v|^{2}}}\right), \quad \text { on } \Omega .
$$

Let $\alpha \in \mathcal{C}^{1}([B,+\infty)), \beta \in \mathcal{C}^{0}([B,+\infty))$ be such that

$$
\begin{gathered}
\alpha(u-v) \geq 0 \\
\alpha^{\prime}(u-v) \geq \beta(u-v)>0,
\end{gathered}
$$

on $\bar{\Omega}_{B}$, and let $\lambda \in \mathcal{C}^{1}(\mathbb{R})$ satisfy

$$
\lambda(t) \geq 0 \text { on } \mathbb{R} ; \quad \lambda(t)=0 \text { on }(-\infty, B] ; \quad \lambda^{\prime}(t) \geq 0 \text { on }(B,+\infty) .
$$

Then, there exists $R_{1}>0$ such that, for each $r>R \geq R_{1}$,

$$
\begin{aligned}
& \frac{1}{\int_{B_{R} \cap \Omega_{B}} \sigma \beta(u-v) \lambda(u-v)\left|\frac{\nabla u}{\sqrt{1+|\nabla u|^{2}}}-\frac{\nabla v}{\sqrt{1+|\nabla v|^{2}}}\right|^{2}} \\
& \geq \int_{R}^{r} \frac{\inf _{\partial B_{s} \cap \Omega_{B}} \sigma}{\int_{\partial B_{s} \cap \Omega_{B}} \lambda(u-v) \frac{\alpha(u-v)^{2}}{\beta(u-v)}} d s .
\end{aligned}
$$

Proof. We keep the notation $\varphi(t)=\left(1+t^{2}\right)^{-\frac{1}{2}}$. Let $Z$ be the vector field defined by

$$
Z=\lambda(u-v) \alpha(u-v)\{\varphi(|\nabla u|) \nabla u-\varphi(|\nabla v|) \nabla v\}, \quad \text { on } \bar{\Omega} .
$$

Note that by our choice of $\lambda$, and the assumption that $\partial \Omega_{B} \subset \Omega, \bar{\Omega}_{B} \subset \Omega$ and $Z \equiv 0$ on $\Omega \backslash \Omega_{B}$. Computing the divergence of $Z$ and using (1.20), 
(1.23), (1.21), (1.22) and the Mikljukov-Hwang-Collin-Krust inequality, we obtain

$$
\operatorname{div} Z \geq \sigma \lambda(u-v) \beta(u-v)|\varphi(|\nabla u|) \nabla u-\varphi(|\nabla v|) \nabla v|^{2} \quad \text { on } \bar{\Omega}_{B} .
$$

Let $s_{0}=\inf \left\{s: B_{s} \cap \Omega_{B} \neq \varnothing\right\}$. Integrating over $\Omega \cap B_{s}$, and applying the divergence theorem and recalling that $Z \equiv 0$ outside $\Omega_{B}$ yield

$$
\int_{B_{s} \cap \Omega_{B}} \sigma \lambda(u-v) \beta(u-v)|\varphi(|\nabla u|) \nabla u-\varphi(|\nabla v|) \nabla v|^{2} \leq \gamma(s)
$$

with

$$
\gamma(s)=\int_{\partial B_{s} \cap \Omega_{B}}\left\langle Z, \frac{\partial}{\partial s}\right\rangle
$$

for $s \geq s_{0}$. Further, Schwarz inequality, the positivity of $\beta(u-v)$ and of $\sigma$, and Hölder inequality give

$$
\begin{aligned}
\gamma(s) \leq & \frac{1}{\left\{\inf _{\partial B_{s} \cap \Omega_{B}} \sigma\right\}^{\frac{1}{2}}}\left\{\int_{\partial B_{s} \cap \Omega_{B}} \lambda(u-v) \frac{\alpha(u-v)^{2}}{\beta(u-v)}\right\}^{\frac{1}{2}} \\
& \cdot\left\{\int_{\partial B_{s} \cap \Omega_{B}} \sigma \lambda(u-v) \beta(u-v)|\varphi(|\nabla u|) \nabla u-\varphi(|\nabla v|) \nabla v|^{2}\right\}^{\frac{1}{2}} .
\end{aligned}
$$

Set

$$
\begin{aligned}
\zeta(s) & =\frac{1}{\left\{\inf _{\partial B_{s} \cap \Omega_{B}} \sigma\right\}^{\frac{1}{2}}}\left\{\int_{\partial B_{s} \cap \Omega_{B}} \lambda(u-v) \frac{\alpha(u-v)^{2}}{\beta(u-v)}\right\}^{\frac{1}{2}} \\
H(s) & =\int_{B_{s} \cap \Omega_{B}} \sigma \lambda(u-v) \beta(u-v)|\varphi(|\nabla u|) \nabla u-\varphi(|\nabla v|) \nabla v|^{2}
\end{aligned}
$$

so that, by the co-area formula

$$
H^{\prime}(s)=\int_{\partial B_{s} \cap \Omega_{B}} \sigma \lambda(u-v) \beta(u-v)|\varphi(|\nabla u|) \nabla u-\varphi(|\nabla v|) \nabla v|^{2} .
$$

From (1.25) and (1.26) we obtain

$$
H(s) \leq \zeta(s)\left(H^{\prime}(s)\right)^{\frac{1}{2}} .
$$

Since our assumptions on $u-v$ and on $\Omega_{B}$ imply that $\nabla(u-v) \neq 0$ on $\Omega_{B}$, we deduce that there exists $R_{1}>0$ such that, for each $s \geq R_{1}$,

$$
H(s)>0 \text {, }
$$

and therefore, by (1.27), $H^{\prime}(s)$ and $\zeta(s)>0$ for $s>R_{1}$. Rearranging we obtain

$$
\frac{H^{\prime}(s)}{H(s)^{2}} \geq \frac{1}{\zeta(s)^{2}}
$$


and then, integrating over $[R, r]$ with $R_{1} \leq R<r$,

$$
\frac{1}{H(R)} \geq \frac{1}{H(R)}-\frac{1}{H(r)} \geq \int_{R}^{r} \frac{d s}{\zeta(s)^{2}}
$$

Recalling the definitions of $H(R)$ and $\zeta(s)$ we obtain (1.24).

Remark. Under the assumption that $\alpha$ and $\beta$ satisfy (1.21) and (1.22) on $M$, the proof of Lemma 1.2 goes through with $M$ in place of $\Omega_{B}$ and with $\lambda \equiv 1$. The conclusion (1.24) is modified accordingly.

Theorem 1.3. Let $\Omega$ be an unbounded domain in $M$, and let $u, v \in \mathcal{C}^{2}(\Omega) \cap$ $\mathcal{C}^{0}(\bar{\Omega})$ be such that

$$
\begin{cases}\operatorname{div}\left(\frac{\nabla u}{\sqrt{1+|\nabla u|^{2}}}\right) \geq \operatorname{div}\left(\frac{\nabla v}{\sqrt{1+|\nabla v|^{2}}}\right) & \text { on } \Omega \\ u \leq v & \text { on } \partial \Omega .\end{cases}
$$

Assume that, for some $q>1$,

$$
\frac{\inf _{\partial B_{s} \cap \Omega} \sigma}{\int_{\partial B_{s} \cap \Omega}|u-v|^{q}} \notin L^{1}(+\infty)
$$

with $\sigma$ as in (1.1). If $\partial \Omega \neq \varnothing$ then $u \leq v$ on $\Omega$. If $\partial \Omega=\varnothing$, i.e., $\Omega=M$, and $u-v$ is not constant, then $u \leq v$.

Remark. If $\Omega$ is bounded, the result is well-known and it holds true regardless of (1.29).

Proof. We consider first the case where $\partial \Omega \neq \varnothing$. Assume by contradiction that

$$
\{x \in \Omega: u(x)>v(x)\} \neq \varnothing .
$$

We choose $B>0$ so small that

$$
\Omega_{B}=\{x \in \Omega: u(x)>v(x)+B\} \neq \varnothing .
$$

Since $u \leq v$ on $\partial \Omega \neq \varnothing, u-v$ is nonconstant on (every connected component of) $\Omega_{B}$ and $\partial \Omega_{B} \subset \Omega$. We apply Lemma 1.2 with the choices

$$
\alpha(s)=s^{q-1}, \quad \beta(s)=(q-1) s^{q-2}, q>1, \quad \text { on }[B,+\infty),
$$

and with $\lambda$ satisfying the further requirement $\sup _{\mathbb{R}} \lambda=1$. According to (1.24), for each $r>R \geq R_{1}>0$, we have

$$
\begin{aligned}
& \frac{1}{\int_{B_{R} \cap \Omega_{B}} \sigma \lambda(u-v)(u-v)^{q-2}\left|\frac{\nabla u}{\sqrt{1+|\nabla u|^{2}}}-\frac{\nabla v}{\sqrt{1+|\nabla v|^{2}}}\right|^{2}} \\
& \geq C \int_{R}^{r} \frac{\inf _{\partial B_{s} \cap \Omega_{B}} \sigma}{\int_{\partial B_{s} \cap \Omega_{B}}(u-v)^{q}} d s \\
& \geq C \int_{R}^{r} \frac{\inf _{\partial B_{s} \cap \Omega} \sigma}{\int_{\partial B_{s} \cap \Omega}|u-v|^{q}} d s,
\end{aligned}
$$


with $C=(q-1)^{2}$. Letting $r \rightarrow+\infty$ this contradicts (1.29).

Next, we consider the case $\partial \Omega=\varnothing$, that is, $\Omega=M$. Again, we argue by contradiction and assume that $\{x \in M: u(x)>v(x)\} \neq \varnothing$ and that $u-v$ is nonconstant on $M$. We choose $B>0$ in such a way that $\Omega_{B}=\{x: u(x)>$ $v(x)+B\}$ is not empty with (possibly empty) boundary $\partial \Omega_{B}$. Note that $u-v$ is nonconstant on every connected component of $\Omega_{B}$. Indeed, this is obvious if $\partial \Omega_{B}=\varnothing$, for then $\Omega_{B}=M$, and follows from the definition of $\Omega_{B}$ otherwise. A contradiction is reached as above, applying Lemma 1.2.

Proof of Theorem B. We apply Theorem 1.3 and use the following chain of implications whose proof is left as an exercise.

$\left\{\begin{array}{l}\text { Let } F:[0,+\infty) \rightarrow[0,+\infty) \text { be such that } \frac{1}{r F(r)} \notin L^{1}(+\infty) \text { and either } \\ \liminf \inf _{r \rightarrow+\infty} \frac{\int_{B_{r} \cap \Omega}|u-v|^{q}}{r^{2}}<+\infty, \quad \text { or } \lim \sup _{r \rightarrow+\infty} \frac{\int_{B_{r} \cap \Omega}|u-v|^{q}}{r^{2} F(r)}<+\infty\end{array}\right.$

$$
\frac{r}{\int_{B_{r} \cap \Omega}|u-v|^{q}} \notin L^{1}(+\infty) .
$$

$$
\frac{1}{\int_{\partial B_{r} \cap \Omega}|u-v|^{q}} \notin L^{1}(+\infty) .
$$

Then (1.30) implies (1.31) which implies (1.32).

The assumption $q>1$ in Theorem 1.3 is sharp. This will be discussed in Section 2. However, for $q=1$, we have the following:

Theorem 1.4. Let $\Omega$ be an unbounded domain in $M$, and let $u, v \in \mathcal{C}^{2}(\Omega) \cap$ $\mathcal{C}^{0}(\bar{\Omega})$ satisfy (1.28). Assume that, for some constants $b, C>0$ and every sufficiently large $r$ we have

$$
\text { i) } \int_{\partial B_{r} \cap \Omega}|u-v| \leq C \frac{1}{r(\log r)^{b}} ; \quad \text { ii) }|u-v| \leq C e^{r(x)^{2}} .
$$

If $\partial \Omega \neq \varnothing$ then $u \leq v$ on $\Omega$. If $\partial \Omega=\varnothing$, that is $\Omega=M$, and $u-v$ is not constant, then $u \leq v$.

Proof. The argument resembles that of Theorem 1.3. We thus consider only the case $\partial \Omega \neq \varnothing$. We apply Lemma 1.2 with

$$
\alpha(s)=\{\log [1+\log (1+s)]\}^{b}, \quad \beta(s)=\frac{b\{\log [1+\log (1+s)]\}^{b-1}}{[1+\log (1+s)](1+s)}=\alpha^{\prime}(s),
$$


on $[B,+\infty)$, and with $\lambda$ satisfying $\sup _{\mathbb{R}} \lambda=1$ to obtain

$$
\begin{aligned}
& \left(\int_{B_{R} \cap \Omega_{B}} \frac{\sigma \lambda(u-v)\left|\frac{\nabla u}{\sqrt{1+|\nabla u|^{2}}}-\frac{\nabla v}{\sqrt{1+|\nabla v|^{2}}}\right|^{2}}{\geq C \int_{R}^{r} \frac{\inf _{\partial B_{s} \cap \Omega_{B}} \sigma d s}{\int_{\partial B_{s} \cap \Omega_{B}}(u-v)[1+\log (1+u-v)]\left\{1+[\log (1+\log (1+u-v))]^{1+b}\right\}}}\right.
\end{aligned}
$$

for $r>R \geq R_{0}$ sufficiently large. Now, using (1.33) i) and ii), we deduce that

$$
\begin{aligned}
& \int_{\partial B_{s} \cap \Omega_{B}}(u-v)[1+\log (1+u-v)]\left\{1+[\log (1+\log (1+u-v))]^{1+b}\right\} \\
& \leq C s \log s,
\end{aligned}
$$

for $s$ large. A contradiction is obtained by taking $r$ sufficiently large in (1.34).

Our next result relates to the conclusion of Theorem 1.3. We stress that we do not assume "a priori" that the graphs $\Gamma_{u}$ and $\Gamma_{v}$, associated to $u$ and $v$, have the same mean curvature.

Theorem 1.5. Let $u, v \in \mathcal{C}^{2}(M)$ be such that

$$
\operatorname{div}\left(\frac{\nabla u}{\sqrt{1+|\nabla u|^{2}}}\right) \geq \operatorname{div}\left(\frac{\nabla v}{\sqrt{1+|\nabla v|^{2}}}\right), \quad \text { on } M \text {. }
$$

Assume that

$$
\frac{1}{\operatorname{vol}\left(\partial B_{r}\right)} \notin L^{1}(+\infty)
$$

If

$$
u \leq v, \quad \text { on } M \text {. }
$$

then, $u=v+A$ on $M$ for some constant $A \leq 0$.

Proof. Assume, by contradiction, that $u-v$ is nonconstant on $\Omega$. The required contradiction follows applying the remark following the proof of Lemma 1.2 with $\alpha(t)=\beta(t)=e^{t}$, and using (1.36) and (1.37).

Because of Condition (1.29), Theorem 1.3 can be naturally regarded as an $L^{q}$-comparison result. In the literature we can find $L^{\infty}$-versions of this result, where (1.29) is replaced by a condition involving $\|u-v\|_{\infty}$. In a sense, Theorem 1.5 goes in this direction. To the best of our knowledge the most general result of this kind is Theorem 3 in [Hw1], which improves on 
Collin and Krust, $[\mathbf{C K}]$, Hwang, $[\mathbf{H w}]$, Mikljukov, $[\mathbf{M}]$, and Langevin and Rosenberg, $[\mathbf{L R}]$. All these results pertain to unbounded domains of $\mathbb{R}^{2}$. As a matter of fact they can be extended to domains in a complete manifold $(M,\langle\rangle$,$) . We briefly describe the argument, which is based on Lemma 1.2$ and on a simple companion estimate. In (1.24) of Lemma 1.2, we obtain an upper bound for the quantity

$$
\int_{B_{R} \cap \Omega_{B}} \sigma \lambda(u-v) \beta(u-v)\left|\frac{\nabla u}{\sqrt{1+|\nabla u|^{2}}}-\frac{\nabla v}{\sqrt{1+|\nabla v|^{2}}}\right|^{2} .
$$

Reasoning in a similar way we can deduce a lower bound of the form

$$
\begin{aligned}
& \int_{\left(B_{r} \backslash B_{R}\right) \cap \Omega_{B}} \sigma \lambda(u-v) \beta(u-v)\left|\frac{\nabla u}{\sqrt{1+|\nabla u|^{2}}}-\frac{\nabla v}{\sqrt{1+|\nabla v|^{2}}}\right|^{2} \\
& \geq C \int_{R}^{r} \frac{\inf _{\partial B_{s} \cap \Omega} \sigma}{\int_{\partial B_{s} \cap \Omega} \beta(u-v) \lambda(u-v)}
\end{aligned}
$$

valid for $r>R \geq R_{2}>0$ sufficiently large and appropriate $\lambda$ and $\beta$.

Combining (1.24) and (1.38) with a suitable choice of $\alpha, \beta, \lambda$, and proceeding as in the proof of Theorem 3 of [Hw1], we obtain the following:

Theorem 1.6. Let $\Omega \subseteq(M,\langle\rangle$,$) be an unbounded domain, and let u, v \in$ $\mathcal{C}^{2}(\Omega) \cap \mathcal{C}^{0}(\bar{\Omega})$ satisfy (1.28). Assume that

$$
\frac{\inf _{\partial B_{r} \cap \Omega} \sigma}{\operatorname{vol}\left(\partial B_{r} \cap \Omega\right)} \notin L^{1}(+\infty)
$$

and that

$$
\liminf _{r \rightarrow+\infty} \frac{\sup _{\partial B_{r} \cap \Omega}(u-v)}{\int_{R}^{r} \frac{\inf _{\partial B_{r} \cap \Omega} \sigma}{\operatorname{vol}\left(\partial B_{r} \cap \Omega\right)} d s}=0,
$$

for some $R>0$ sufficiently large. If $\partial \Omega \neq \varnothing$, then, $u \leq v$ on $\Omega$. If $\Omega=M$, and $u-v$ is not constant, then $u \leq v$.

Remark. Replacing (1.28) with (0.3) and (1.39) with

$$
\liminf _{r \rightarrow+\infty} \frac{\sup _{\partial B_{r} \cap \Omega}|u-v|}{\int_{R}^{r} \frac{\inf _{\partial B_{r} \cap \Omega} \sigma}{\operatorname{vol}\left(\partial B_{r} \cap \Omega\right)} d s}=0,
$$

Theorem 1.6 yields a uniqueness result which extends to manifolds Theorem 3 of $[\mathbf{H w} \mathbf{1}]$ and Théorème 2 of $[\mathbf{C K}]$. 


\section{Some examples.}

In this section we describe examples showing that Theorems A and 1.3 are fairly sharp.

Towards this aim, let $g(r) \in \mathcal{C}^{\infty}([0,+\infty))$ be such that

i) $g(r)>0$ for $r>0$; $\quad$ ii) $g^{\prime}(0)=1$; $\quad$ iii) $g^{(2 k)}(0)=0$, for each $k=0,1, \ldots$ On $\mathbb{R}^{m} \backslash\{0\}=(0,+\infty) \times S^{m-1}, m \geq 2$, we define the metric

$$
\langle,\rangle=d r^{2}+g(r)^{2} d \theta^{2}
$$

with $d \theta^{2}$ the standard metric on $S^{m-1}$. Because of (2.1) ii), iii) we can extend $\langle$,$\rangle to a smooth, obviously complete, metric on \mathbb{R}^{m}$. Given a function $a(r) \in \mathcal{C}^{\infty}([0,+\infty))$, even at 0 , that is, $a^{(2 k+1)}(0)=0, k=0,1,2, \ldots$, we let

$$
h(t)=g(t)^{1-m} \int_{0}^{t} g(y)^{m-1} a(y) d y .
$$

If

$$
h(t)<1, \quad \text { on }[0,+\infty)
$$

we may define

$$
\beta(r)=\beta_{0}+\int_{0}^{r} \frac{h(t)}{\sqrt{1-h(t)^{2}}} d t, \quad \beta_{0} \in \mathbb{R},
$$

and the function

$$
u(x)=\beta(r(x))
$$

is smooth on $\mathbb{R}^{m}$ and defines a graph $\Gamma_{u}$ with mean curvature $\frac{1}{m} a(r(x))$.

For the sake of simplicity, in what follows we consider the case $m=2$.

The first examples refer to Theorem A. We begin by showing that the boundedness of $u$ is necessary to conclude.

(1) Let $g(r)=r$ on $[0,+\infty)$. Since $m=2$,

$$
\operatorname{vol}\left(\partial B_{r}\right)=2 \pi r
$$

and (i) holds with $\alpha=1, C=1$. Let

$$
a(r)=\frac{1}{\left(e+r^{2}\right) \log \left(e+r^{2}\right)} \sim \frac{1}{2 r^{2} \log r}, \quad \text { as } r \rightarrow+\infty,
$$

so that (j) is not satisfied. With the previous notations

$$
h(t)=\frac{1}{2 t} \log \log \left(e+t^{2}\right),
$$

and it is a calculus exercise to verify that (2.3) is satisfied. An easy computation shows that $u(x) \sim[\log r(x)][\log \log r(x)]$, as $r(x) \rightarrow+\infty$, and (0.1) is not satisfied. 
(2) We choose $g(r)=r e^{\sqrt{1+r^{2}}-1}$. It is readily seen that requirements $(2.1)$ are satisfied.

$$
\operatorname{vol}\left(\partial B_{r}\right) \sim 2 \pi e^{-1} r e^{r}, \quad \text { as } r \rightarrow+\infty
$$

and it satisfies (ii) for any $\alpha>1, C>0$ sufficiently large. Let

$$
a(r)=\frac{1}{\sqrt{1+r^{2}}} \sim \frac{1}{r}, \quad \text { as } r \rightarrow+\infty,
$$

so that (jj) is not satisfied. Next,

$$
h(t)=\frac{e^{\sqrt{1+t^{2}}-1}-1}{t e^{\sqrt{1+t^{2}}-1}},
$$

and one checks that (2.3) is satisfied and $h(t) \rightarrow 0$, as $t \rightarrow+\infty$. Here $u(x) \sim \log r(x)$, as $r(x) \rightarrow+\infty$, and (0.1) is not satisfied.

(3) Let $g(r)=r e^{r^{2}}$. Then Conditions (2.1) are met, and since

$$
\operatorname{vol}\left(\partial B_{r}\right)=2 \pi r e^{r^{2}}
$$

(iii) is satisfied for $\alpha>1, C=2 \pi$. Let $a(r) \equiv 1$. so that (jjj) is not satisfied and

$$
h(t)=\frac{1}{2} \frac{e^{t^{2}}-1}{t e^{t^{2}}} .
$$

Again, (2.3) is satisfied and since $u(x) \sim \log r(x)$, as $r(x) \rightarrow+\infty,(0.1)$ is not met.

The next example shows that the volume growth conditions in the statement of the theorem are almost optimal. We consider only the growth Condition (iii), leaving the remaining cases to the interested reader.

(4) Let $g(r)=\frac{1}{2} \frac{d}{d r} e^{r^{2}\left\{1+\left[\log \left(1+r^{2}\right)\right]^{2}\right\}}$. Clearly, (2.1) holds. Since

$$
\operatorname{vol}\left(\partial B_{r}\right) \sim C r e^{4 r^{2}(\log r)^{2}}(\log r)^{2}, \quad \text { as } r \rightarrow+\infty,
$$

for some constant $C>0$, (iii) is not satisfied for any choice of $\alpha>0$. In this case, we choose $a(r) \equiv 1$ so that (jjj) fails. Furthermore,

$$
h(t)=\frac{1}{2} g(t)^{-1}\left[e^{t^{2}\left\{1+\left[\log \left(1+t^{2}\right)\right]^{2}\right\}}-1\right] \sim \frac{C}{t(\log t)^{2}}, \quad \text { as } t \rightarrow+\infty .
$$

Thus, $h(t) \rightarrow 0$ as $t \rightarrow+\infty$ and $h(t) \in L^{1}(+\infty)$. Again a calculus exercise shows the validity of (2.3). Furthermore, $u(x)=\beta(r(x))$ is nonnegative and bounded above, so that (0.1) is satisfied.

The next three examples show that the decay rate conditions for $a$ in Theorem A are almost optimal. 
(5) We fix any $\mu>0$ and we set $g(r)=r\left[\log \left(e+r^{2}\right)\right]^{1+\frac{\mu}{2}}$. Clearly, (2.1) is satisfied. Also,

$$
\operatorname{vol}\left(\partial B_{r}\right) \sim \pi 2^{2+\frac{\mu}{2}} r(\log r)^{1+\frac{\mu}{2}}, \quad \text { as } r \rightarrow+\infty
$$

so that Condition (i) holds true. We let

$$
a(r)=\frac{C_{0}}{\left(e+r^{2}\right)\left[\log \left(e+r^{2}\right)\right]^{2+\mu}},
$$

where $C_{0}>0$ is a suitable constant. A computation shows that

$$
h(t)=\frac{C_{0}}{\mu} \frac{1-\left[\log \left(e+t^{2}\right)\right]^{-\frac{\mu}{2}}}{t\left[\log \left(e+t^{2}\right)\right]^{1+\frac{\mu}{2}}},
$$

and we can choose $C_{0}>0$ so small that (2.3) is met. Moreover, since $h(t) \rightarrow 0$, as $t \rightarrow+\infty$ and $h(t) \in L^{1}(+\infty)$ we have

$$
\beta_{0} \leq u \leq \sup _{\mathbb{R}^{2}} u<+\infty, \quad \beta_{0} \in \mathbb{R} .
$$

Condition (0.1) is satisfied.

(6) Let $g(r)=r e^{\sqrt{1+r^{2}}-1} \log \left(e+r^{2}\right)\left[\log \left(\log \left(e^{2}+r^{2}\right)\right)\right]^{2}$, so that both (2.1) and the volume growth Condition (ii) are satisfied. Let also

$$
a(r)=\frac{1}{\sqrt{1+r^{2}} \log \left(e+r^{2}\right)\left[\log \left(\log \left(e^{2}+r^{2}\right)\right)\right]^{2}}
$$

and note that

$$
\lim _{r(x) \rightarrow+\infty} \frac{a(r(x))}{\left\{r(x) \log r(x)[\log (\log r(x))]^{2}\right\}^{-1}}>0 .
$$

A computation shows that

$$
h(t)=\frac{1-e^{-\sqrt{1+t^{2}}+1}}{t \log \left(e+t^{2}\right)\left[\log \left(\log \left(e^{2}+t^{2}\right)\right)\right]^{2}} .
$$

Thus, (2.3) is satisfied. Furthermore, $h(t) \rightarrow 0$ as $t \rightarrow+\infty$ and $h(t) \in$ $L^{1}(+\infty)$, so that,

$$
\beta_{0} \leq u \leq \sup _{\mathbb{R}^{2}} u<+\infty, \quad \beta_{0} \in \mathbb{R},
$$

and Condition (0.1) holds.

(7) Let $g(r)=r e^{r^{2}} \log \left(e+r^{2}\right)\left[\log \left(\log \left(e^{2}+r^{2}\right)\right)\right]^{2}$, so that (2.1) and the volume growth Condition (iii) are satisfied, and define

$$
a(r)=\frac{1}{\log \left(e+r^{2}\right)\left[\log \left(\log \left(e^{2}+r^{2}\right)\right)\right]^{2}}
$$


so that

$$
\lim _{r(x) \rightarrow+\infty} \frac{a(r(x))}{\left\{\log r(x)[\log (\log r(x))]^{2}\right\}^{-1}}>0 .
$$

A computation shows that

$$
h(t)=\frac{1-e^{-t^{2}}}{t \log \left(e+t^{2}\right)\left[\log \left(\log \left(e^{2}+t^{2}\right)\right)\right]^{2}},
$$

so that $(2.3)$ holds true. Moreover $h(t) \rightarrow 0$ as $t \rightarrow+\infty$ and $h(t) \in L^{1}(+\infty)$ and therefore $u$ is bounded.

We now consider Theorem 1.3.

(1) Let $(M,\langle\rangle)=,\left(\mathbb{R}^{2}\right.$, can $), \Omega=\mathbb{R}^{2} \backslash B_{1}(0), v(x) \equiv 0, u(x)=\operatorname{arccosh} r(x)$. Then, $\Gamma_{u}$ is minimal on $\bar{\Omega}, u(x) \equiv v(x) \equiv 0$ on $\partial \Omega$. Since $\operatorname{arccosh} r \sim \log r$ as $r \rightarrow+\infty$, for any $q>1$ there exists $r \gg 1$ and $C>0$ such that

$$
\frac{1}{\int_{\partial B_{r} \cap \Omega}|u-v|^{q}} \leq \frac{C}{r(\log r)^{q}} \in L^{1}(+\infty) .
$$

Thus, (1.29) is not satisfied. Here, $\partial \Omega \neq \varnothing$ but $u \leq v$ on $\Omega$ is false.

(2) Let $g(r) \in \mathcal{C}^{\infty}([0,+\infty)), g(r)>0$ for $r>0, g^{\prime}(r) \geq 0$ and

$$
g(r)=\left\{\begin{array}{ll}
r & \text { on }\left[0, \frac{1}{2}\right] \\
r^{\eta-1} & \text { on }[1,+\infty)
\end{array}, 1 \leq \eta<2,\right.
$$

so that

$$
\operatorname{vol}\left(\partial B_{r}\right)=2 \pi r^{\eta-1} \text {. }
$$

Let also $a(r) \in \mathcal{C}^{\infty}([0,+\infty))$, be such that $a(r) \geq 0$ and

$$
a(r)=C \begin{cases}1 & \text { on }[0,1] \\ 0 & \text { on }(2,+\infty) .\end{cases}
$$

By taking $C$ sufficiently small we may arrange that, having defined $h(t)$ as in (2.2), (2.3) holds. Then the function $u(x)=\beta(r(x))$ defined by (2.4) is smooth on $\mathbb{R}^{2}$, and defines a graph with nonnegative mean curvature (equal to $a(x) / m)$. It is easily verified that

$$
u(x) \sim C r(x)^{2-\eta},
$$

for some $C>0$. Thus, if $v \equiv 0$, for each $q>1$ we have

$$
\frac{1}{\int_{\partial B_{r}}(u-v)^{q}} \leq \frac{C}{r^{q(2-\eta)+\eta-1}} \in L^{1}(+\infty) .
$$

Here, $\partial \Omega=\varnothing$ since $\Omega=\mathbb{R}^{2}$. We also note, in this last example, that

$$
\frac{1}{\int_{\partial B_{r}}(u-v)} \notin L^{1}(+\infty)
$$


so that, Condition (1.29) in Theorem 1.3 cannot be improved to $q \geq 1$.

We would like to conclude with the following observation. In a recent paper, Alencar and do Carmo, $[\mathbf{A d C}]$, prove that if $f: M \rightarrow(N, h)$ is an isometrically immersed complete, noncompact hypersurface with constant mean curvature and of at most polynomial growth, then $f$ is minimal provided $\operatorname{Ricc}_{(N, h)} \geq 0$ and a certain operator has finite index (graphs satisfy this property).

It seems an interesting problem to extend Theorem A in this direction.

\section{References}

$[\mathrm{AdC}] \mathrm{H}$. Alencar and M. do Carmo, Hypersurfaces of constant mean curvature with finite index and volume of polynomial growth, Arch. Math., 60 (1993), 489-493; Erratum, ibid., 65 (1995), 271-272, MR 94a:53087, Zbl 0787.53055; MR 96e:53071, Zbl 0833.53051.

[B1] S. Bernstein, Sur la généralisation du problème de Dirichlet, Math. Ann., 69 (1910), 82-136.

[B2] Sur les surfaces définies au moyen de leur courbure moyenne ou totale, Ann. Sci. Ecole Norm. Sup., 27 (1910), 233-256.

[C] S.S. Chern, On the curvature of a piece of hypersurface in Euclidean space, Abh. Math. Sem. Univ. Hanburg, 29 (1965), 77-91, MR 32 \#6376, Zbl 0147.20901.

[CK] P. Collin and R. Krust, Le problème de Dirichlet pour l'equation des surfaces minimales sur des domaines non bornés, Bull. Soc. Math. France, 119 (1991), 443-458, MR 92m:53007, Zbl 0754.53013.

[F] R. Finn, Remarks relevant to minimal surfaces, and to surfaces of prescribed mean curvature, J. Analyse Math., 14 (1965), 139-160, MR 32 \#6337, Zbl 0163.34604.

[G] A. Grigor'yan, On stochastically complete manifolds (in Russian), DAN SSSR, 290 (1986), 534-537; Engl. transl. in Soviet Math Dokl., 34 (1987), 310-313, MR 88a:58209, Zbl 0632.58041.

[G1] - Bounded solutions of the Schrödinger equation on noncompact Riemannian manifolds (in Russian), Trudy seminara I.G. Petrovskogo, 14 (1989), 6677; Engl. transl. Journal of Soviet Math., 51 (1990), 2340-2349, MR 90m:35050, Zbl 0744.58070.

$[\mathrm{H}] \quad$ E. Heinz, Über Flächen mit eineindeutiger Projektion auf eisse Ebene, deren krümmungen durch Ungleichungen eingeschränkt sind, Math. Ann., 129 (1955), 451-454, MR 17,189a, Zbl 0065.37201.

[Hw] J.F. Hwang, Comparison principles and theorems for prescribed mean curvature equation in unbounded domains, Ann. Scuola Norm. Sup. Pisa, 15(4) (1988), 341355, MR 90m:35019, Zbl 0705.49022.

[Hw1] _ A uniqueness theorem for the minimal surface equation, Pacific J. Math., 176 (1996), 357-364, MR 97m:53010, Zbl 0870.53005.

[LR] R. Langevin and H. Rosenberg, A maximum principle at infinity for minimal surfaces and applications, Duke Math. J., 57 (1988), 819-828, MR 90c:53025, Zbl 0667.49024. 
[M] V.M. Mikljukov, A new approach to Bernstein theorem and to related questions for equations of minimal surface type (in Russian), Mat. Sb. 108 (1979), 268-289; Engl. transl. in Math. USSR Sb., 36 (1980), 251-271, MR 80e:53005.

[RSV] M. Rigoli, M. Salvatori and M. Vignati, A Liouville type theorem for a general class of operators on complete manifolds, Pacific J. Math., 194 (2000), 439-453, MR 2001g:58061.

[S] I.C.M. Salavessa, Graphs with parallel mean curvature, Proc. Amer. Math. Soc., 107 (1989), 449-458, MR 90a:53072, Zbl 0681.53031.

Received December 28, 2000 and revised May 14, 2001.

Dipartimento di Matematica

UNiVERSità Di Milano

VIA SALDINI 50

I-20133 Milano

ITALY

E-mail address: pigola@mat.unimi.it

Dipartimento di Scienze C.F.M.

UNIVERSitÀ DELL'INSUBRIA - COMO

via Valleggio 11, I-22100 Como

ITALY

E-mail address: rigoli@matapp.unimib.it

Dipartimento di Scienze C.F.M.

UNIVERSitÀ DELL'INSUBRIA - COMO

Via VAlleggio 11

I-22100 Como

ITALY

E-mail address: setti@uninsubria.it 